\title{
E. New Techniques
}

\section{HEART RATE CONTROLLED ERGOMETRY}

\author{
L. G. EKELUND, M.D. \\ Dept. of Clinical Physiology, Karolinska sjukhuset, S 10401 Stockholm, Sweden.
}

\begin{abstract}
The control system of an electrically braked ergometer (Elema corp.) has been changed in order to include the possibility to obtain an arbitrarily chosen heart rate automatically. The control unit consists of a heart rate meter triggered by ECG and a servosystem which is constructed to give a smooth load and heart rate response and to hold the present heart rate within about \pm 3 beats $/ \mathrm{min}$. There are analog signals for recording of the heart rate, load and integrated load. The practical applications will be discussed and demonstrated.
\end{abstract}

\section{Introduction}

In ergometry some kind of mechanically or electrically braked cycle is one of the best tools. There are a great number of different modes of using the ergometer. For instance it can be used with a single-level-load, a discontinuous series of increasing loads or a continuous series of increasing loads with a nearly steady state at each level. In Sweden the stepwise increased test of the continuous type is the most common and gives a very good opportunity to study ECG reactions and circulatory variables when investigating patients in whom coronary insufficiency and heart failure is suspected. In such a test one chooses steps of loads in relation to the patient's physical activity and condition. Loads of 200,400 and $600 \mathrm{kpm} / \mathrm{min}$ for females and 300, 600 and $900 \mathrm{kpm} / \mathrm{min}$ for men are

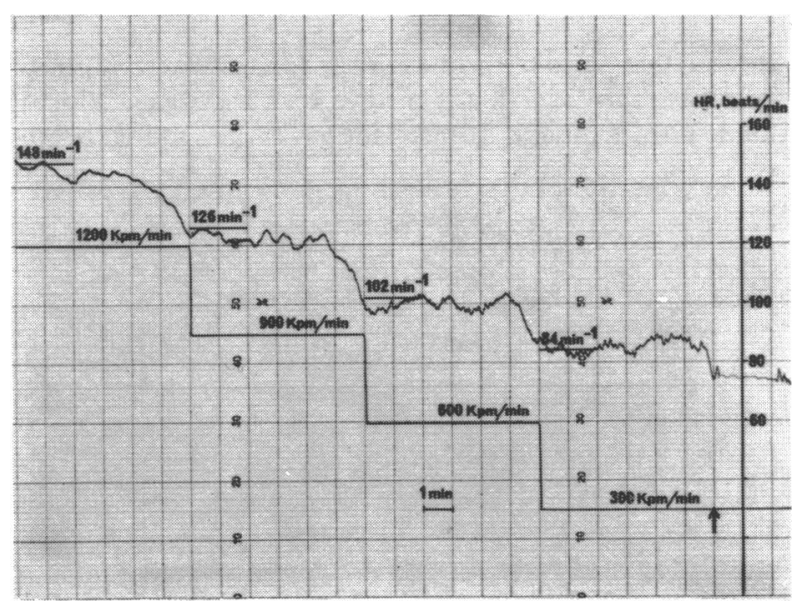

Fig. 1 illustrates the ordinary exercise test with stepwise continuous increasing loads with registration of the heart rate between 5.6 min at each load. These and the following recordings should be read from the right to the left. standard loads for testing normal individuals (fig. 1). However, when a patient is retested after some kind of treatment, it is found that the pulse rate at the preselected loads differs from time to time when fixed loads are used. In this case it would be better to use a heart rate controlled ergometer with which specific heart rates could be selected. A patient could then be tested at the same heart rate each time, for example 110, 130, 150 and 170 beats/min. In this way one could compare the ECG reaction at the same heart rate both before and after some kind of treatment. Also in ordinary fitness testing when the work load giving a heart rate of 170 beats/min $\left(W_{170}\right)$ is used as an index of the oxygen transport capacity of the circulatory system, there would be an advantage in selecting the heart rates instead of having preselected work loads. In the ordinary preselected load type of testing one has to inter- or extrapolate the heart rate to obtain the load which gives a heart rate of 170 beats $/ \mathrm{min}$.

My own special interest in heart rate controlled ergometry is based on my studies of prolonged continuous exercise on a fixed load. Under these conditions there is a continuous increase in heart rate from, for instance, 140 beats $/ \mathrm{min}$ after $10 \mathrm{~min}$ exercise to $170-180$ beats $/ \mathrm{min}$ after $60 \mathrm{~min}$ exercise. These changes were found to reflect a decrease in stroke volume with an unchanged cardiac output. The increase in heart rate over a fixed period is a measure of the unsteady state condition, but one could also have a constant heart rate and measure the corresponding decrease in work load over a fixed period.

Another application is in metabolic studies when exercise is often used. Heart rate is a good index of the relative stress of the exercise but with an ordinary ergometer one has to perform several pilot tests to get the same heart rates for different individuals in order to have them comparable with respect to the relative work 
load. In such cases there is no problem with the heart rate controlled ergometer.

I will now describe briefly the technical details. There are no special technical problems in building a control unit, which changes the load in an electrically braked ergometer in response to a change in heart rate. The problems are of a biological type. One problem is that each individual has his own response to a stepwise increase or decrease in load. To get the ideal response one must, therefore, know each individual's response curve. Another problem is that some individuals have an alinear response with a marked time delay. In the bicycle developed in co-operation with Elema-Schönander in Sweden we have used a control unit which gives an error signal to the servo motor proportional to the deviation in heart rate from the preselected heart rate and also takes into consideration the rate of increase in heart rate, i.e. the first derivate of heart rate (fig. 2). This influence of the rate of increase in heart rate differs both for different levels of heart rate and also for increases or decreases in heart rate (fig. 3). After testing many individuals we have found a rather good optimization of amplification, damping and so on, so that we can obtain the preselected heart rate without a large overshoot in load and have a stable heart rate for prolonged periods of time (fig. 4), within $2-3$ beats/min.

Analogue signals for recording of work load and heart rate are available. The integrated work load, i.e. total work, is indicated on a counter and there is an output for recording of every 10 th $\mathrm{kpm}$.

With this ability to control the heart rate over a prolonged period one can get work load curves with respect to time, which slope indicates the possibility of maintaining a steady state. It is also very easy to produce

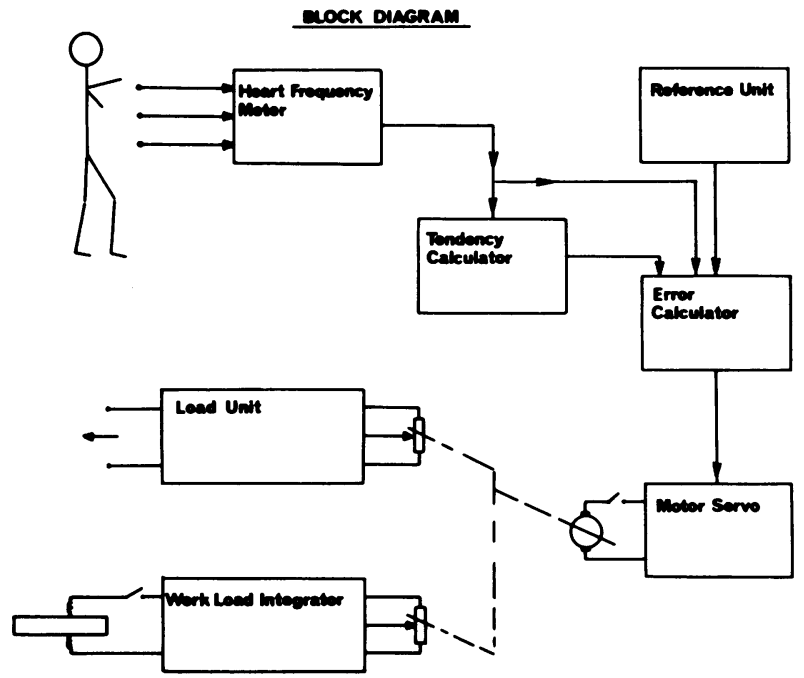

Fig 2 Block diagram over the heart rate controlled ergometer.

\begin{tabular}{|c|c|c|}
\hline \multirow{2}{*}{$\begin{array}{l}\text { Frequoncy } \\
\text { beata/min }\end{array}$} & \multicolumn{2}{|c|}{$\begin{array}{l}\text { The rate of change in froquency which gives the same error slonal } \\
\text { as a frequency deviation of } 10 \text { beats } / \mathrm{min}\end{array}$} \\
\hline & $\begin{array}{l}\text { Rate of increase in frequency } \\
\text { beats } / \min / \min \end{array}$ & $\begin{array}{l}\text { Rate of docrease in frequency } \\
\text { boats } / \mathrm{min} / \mathrm{min}\end{array}$ \\
\hline $\begin{array}{r}>110 \\
110-130 \\
150-150 \\
150-170 \\
<\pi 0\end{array}$ & $\begin{array}{l}90 \\
45 \\
30 \\
22,5 \\
18\end{array}$ & $\begin{array}{l}90 \\
90 \\
90 \\
90 \\
90\end{array}$ \\
\hline
\end{tabular}

Fig. 3. The values for the influence of the first derivate of heart rate.

a standardized work test with preselected heart rates as mentioned earlier (fig. 5) and also to get reproducable heart rates in order to test the ECG reaction after treatment with beta-blocking substances for instance.

A heart rate controlled ergometer is also an excellent instrument with which to train patients, if one wishes to use continuous exercise as a form of training. One can select an appropriate level of heart rate for training, for instance 140 beats $/ \mathrm{min}$, and have the patient work for half an hour 2-3 times a week. I have used the cycle to train medical students at two different heart rates, 130 and 160, for three half-time periods per week with a significant increase in total work output after 6-8 weeks training (fig. 6). This type of training is especially suitable for training patients with coronary insufficiency or post-muocardial infarction patients, where one could very simply select a heart rate which is somewhat below the level which gives anginal pain or undesirable ECG reactions. At present I have only trained four patients who have had cardiac infarctions, but my preliminary impression is very good. For instance one patient who was trained at a heart rate of 140 beats $/ \mathrm{min}$, achieved an increase of 30 per cent in total work output for $30 \mathrm{~min}$.

When testing large populations, for example military conscripts, the time spent on the test is critical, and to obtain our ordinary fitness index $\left(W_{170}\right)$ one often

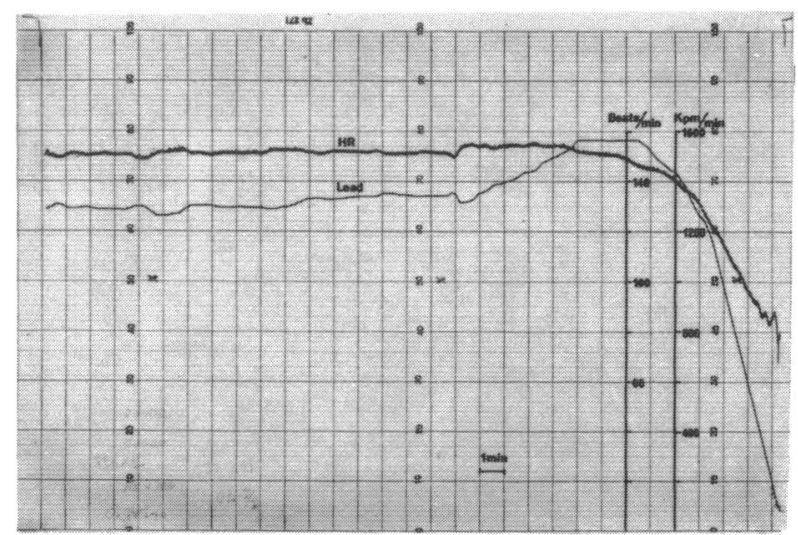

Fig 4. Reconding from a 30 min exercise period. 


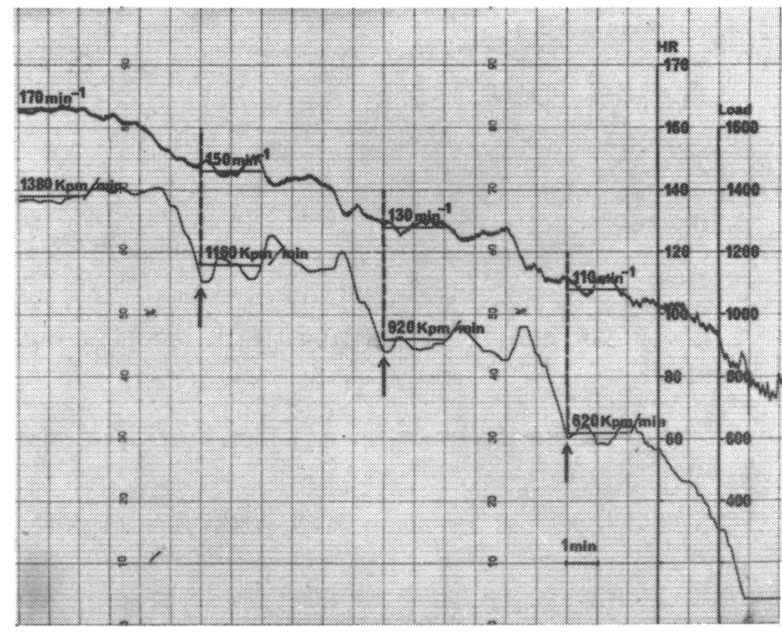

Fig. 5. Recording from an exercise test in the same individual as in fig. 1 but with preselected heart rates.

needs 18-24 $\mathrm{min}$ of exercise. One-load testing has several disadvantages and therefore I have used the heart rate controlled cycle to get a rapid value for the load which gives 170 beats/min (fig. 7). The value after $8 \mathrm{~min}$ is identical with the conventionally obtained value. However, it is important to stress that such a test is only a fitness test which gives no opportunity to study ECG reactions and other variables.

To summarize, there are several valuable applications of a heart rate controlled ergometer:

Rapid fitness testing.

Reproducable ECG studies during exercise when testing the influence of a treatment.

Metabolic studies during exercise.

Bicycle training, especially in coronary patients.

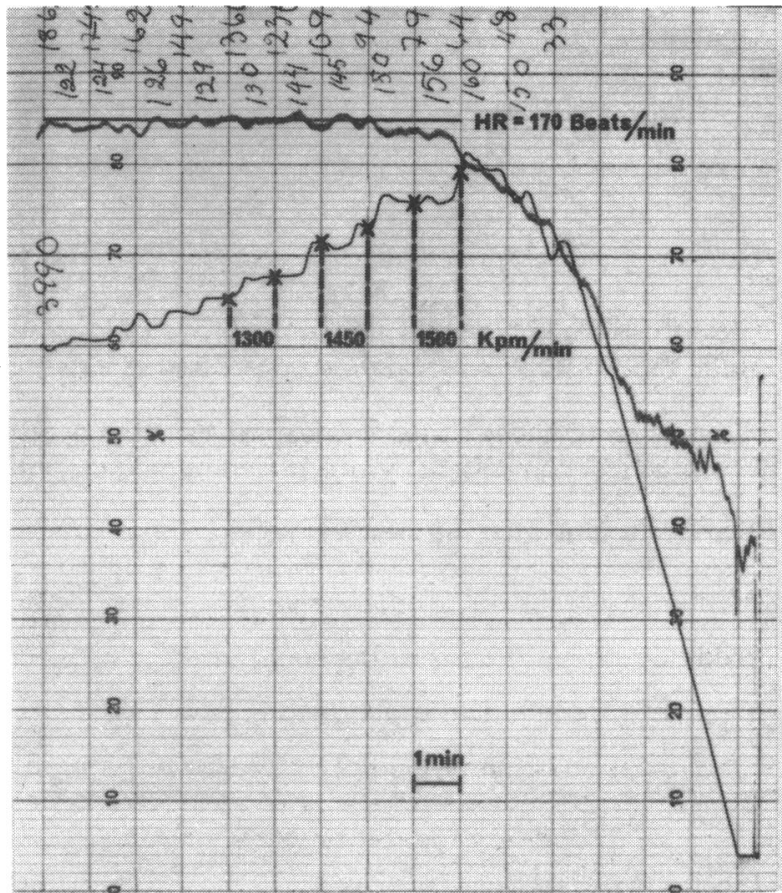

Fig. 6. Reconding from a subject exercising for $60 \mathrm{~min}$ at a heart rate of 150 beats/min. Lower load curve is obtained before training with a total work output of $54160 \mathrm{kpm}$ and the upper load curve after 8 weeks training with a total work output of $68660 \mathrm{kpm}$.

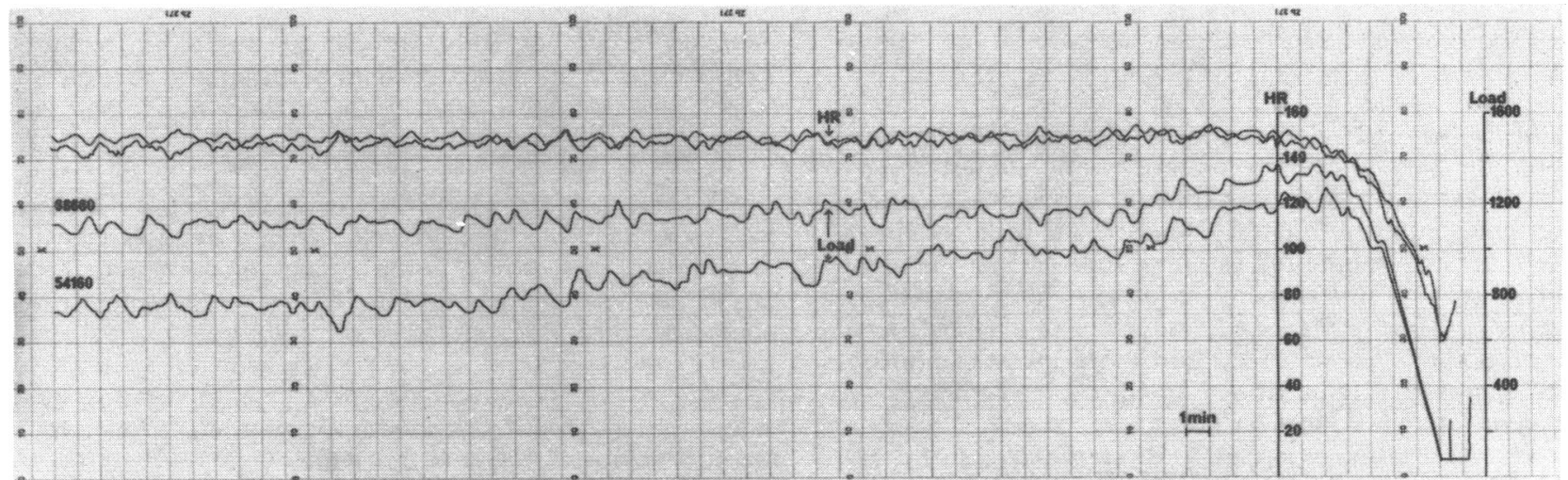

Fig. 7. Rapid fitness test with estimation of the work load giving a heart rate of 170 beats $/ \mathrm{min}\left(\mathrm{W}_{1} 70\right)$, in this case $1450 \mathrm{kpm} / \mathrm{min}$. 


\title{
APPLICATION OF CYBERNETICS TO SPORTS MEDICINE
}

A. F. MATESANZ, M.D., and M. A. GONZALES, M.D.

University of Madrid, Spain

\begin{abstract} Physical Education will be exposed considered as a mathematical science. and its practical applications to the different medical, education and sports levels.

The study is divided into the following items:-

1. Psycho-physical study of the individual.

2. Study of integrant parts of the equation.

3. Study of parameters of sport gesture.

4. Study of sports from the point of view of its biological application.

5. Sport selection.

6. Sport therapeutics.

7. Prevention of sport accident.

8. Programming in the IBM-7090 of the Calculation Centre of the University of Madrid.

9. Summary and conclusions.
\end{abstract}

Classification, system and phenomenology of data inserted in a theoretical pattern comprising Sport, Medicine and

We give some ideas about the way of calculating an equation, its possible programming in an electronic computer

La Educación Física, ciencia joven aún, resulta todavía eminentemente empírica, fraccionada en cuanto a los resultados $y$ aún por coordinar en cuanto a sus componentes.

Se tienen múltiples datos y se hace necesario clasificarlos, ordenarlos, insertarlos al fin en una teoria amplia y general que abarque el desarrollo integral de la persona humana, tanto en su aspecto fisico como educativo.

La ciencia ha alcanzado su plenitud, su nombre propio de ciencia, cuando los fenómenos que estudia se pueden expresar matemáticamente, es decir, se puede crear un modelo mental isomorfo con la realidad exterior. Naturalmente es imposible llegar a este isomorfismo de una manera perfecta, pero nos podemos ir aproximando cada vez más. Este es el verdadero progreso de la ciencia y la constante investigación de profesores y educadores, médicos, psicólogos y pedagogos tiende a alcanzar esta meta.

La Educación Física, como ciencia, dista mucho aún de esta meta. Ha de recorrerse un largo camino. Quizá lo más dificil sea insertar tantos resultados parciales dentro de una teoría general que recoja todos los aspectos de educación integral del hombre. No debemos olvidar que todo, circunstancias del EGO y del NO-EGO, influyen de una manera u otra en todas las acciones humanas. Por tanto en los resultados deportivos entran muchísimos factores que a veces nos parecen extraños y que sin embargo tienen decisiva importancia.

En el presente trabajo nos referimos sobre todo a los deportes como capítulo integrante de la Educación Física y desde un punto de vista práctico a la selección terapéutico-deportiva, a través de los datos morfológicos y funcionales de cada individuo.

La técnica de los años 70 ha dado al hombre nuevos medios para la investigación científica y después nueva posibilidad para una rápida solución de complejos problemas matemáticos inherentes a la investigación operativa.

En la incesante sucesión de inventos $y$ descubrimientos, la compañía del cálculo electrónico ha revolucionado las técnicas corrientes y las indicaciones de este medio autoritario han aumentado rápidamente.

La Medicina y la Biología únicamente en los sectores cientificos y sus enlaces, dependen hoy en dia, más, de 
los nuevos sistemas de simulación que permiten extraer de los complejos fenómenos naturales, los aspectos de mayor relieve e interés tranformándos en términos numéricos.

La teoría de Einstein afirmaba que la comprensión de los hechos naturales puede entenderse por medio de construcciones puramente matemáticas. Los diversos y complejos problemas que manan de la unión de estas ciencias, hoy pueden ser resueltos por el auxilio de las computadoras o calculadores electrónicos.

Tenemos por tanto, justificada la tentativa de acoplar las técnicas de la investigación operativa, es precisamente la teoría de los gráficos y el sistema del PERT en el estudio de los temas inherentes a los problemas del deporte, la que propone continuamente nuevos campos de investigación frontal en la siempre intensa selección del cuerpo humano en el trabajo agonístico.

La teoría de los gráficos se publicó por primera vez en la memoria de Leonard Euler en el año 1763, pudiendo ser subdividida en dos direcciones, una de carácter preferentemente teórico-matemático y otra preferentemente operativa. La segunda dirección es la que nos interesa en la investigación operativa $y$ viene caracterizada en la línea max́ima de la investigación de algoritmos o procedimiento de cálculos hachos para resolver en casos concretos y desde el punto de vista númerico los varios problemas inherentes a aplicaciones diversas.

Es preciso manejar centenares de datos, otras tantas variables que pueden influir en los éxitos o fracasos deportivos, relacionarlos entre sí por medio de los resultados empíricos obtenidos y elaborar una ecuación que nos de aunque sea de un modo aproximado la capacidad deportiva de cada sujeto.

Hace unos años esta labor, por ardua, compleja y extensa, era prácticamente imposible realizarla. Hoy gracias al desarrollo de las computadoras electrónicas es posible y esperamos, aunque sea para un futuro no muy lejano, alcanzar los frutos deseados. Hablar de computadoras y selección de portiva no es una utopía, ni ganas de querer complicar lo fácil, es simplemente la facilidad de organizar la selección deportiva en la Educación Física a diferentes escales. Recordemos que en algunas naciones existe ya un diagnóstico médico dado por ordenadores electrónicos. No olvidemos, sin embargo, que la máquina es una ayuda del hombre y es éste el que tiene que dar la última palabra. No se trata, pues, de eliminar al profesor o al médico en la Educación Física sinó de proporcionarle un valioso instrumento de trabajo. al poder manejar de una manera global muchos más datos de cada persona.

Nuestra finalidad, es pues, crear un modelo matemático que explique el complejo mundo de la selección deportiva, programarlo en el centro de cálculo de la Universidad de Madrid e ir dando a conocer a la sociedad los resultados obtenidos.

Esto será una albor de equipo, y ya desde aqui queremos dar entrada a Organismos Federativos, Pedagogos, Médicos, Psicólogos y todo tipo de Entidades Deportivas en una labor funcional que comienza desde estas líneas directrices del programa. Es una labor de todos, pues a todos nos debe preocupar el bienestar físico de cada persona y como resultado el bienestar y salud de las naciones y de la humanidad entera.

¿La teoría general de los deportes es una ciencia matemática? Nosotros nos atrevemos a decir que sí. La estadística ya ha entrado en todas las ramas del saber. ¿Por qué en ésta no?

Lo que pretendemos es muy sencillo de expresar, no tanto de lograr. Es: "Buscar una ecuación matemática que nos relacione cada deporte con los datos personales o variables deportivas de cada persona".

En adelante la ecuación será designada por:

$$
\begin{aligned}
& D=f\left(x_{1} x_{2} \ldots \ldots, x_{n}\right) . \\
& \text { donde } D=\text { Cada uno de los deportes } \\
& \text { Cada uno de los índices } \\
& \text { deportivos } \\
& \text { Cada uno de los indices } \\
& \text { médico-deportivos, etc. } \\
& f=\quad \text { Función de relación entre } \\
& \text { deportes y datos. }
\end{aligned}
$$

$x_{1}, x_{2}, \ldots, x_{n}=$ Datos personales 0 variables deportivas.

Con un ejemplo: El fútbol ¿cómo está relacionado con el peso, talla, velocidad, resistencia, reflejos, agilidad, coordinación neuromuscular etc.? ¿No sería posible matematizar esta situación? Es decir, a través de los datos personales ino habrá posibilidad de seleccionar para este deporte a los mejores, en posibilidades en un conjunto de alumnos?

Alguién se preguntará: ¿Es posible tener en cuenta tantas variables, manejar tantos datos, en una palabra llegar a obtener esta ecuación?

Nuestro propósito es dar algunas ideas de como calcular la fórmula $D=f(x)$; su posible programación en un ordenar y sus aplicaciones prácticas a nivel local (Colegios, Institutos, Universidades), provincial, regional o nacional. 


\section{FUNCIONES DEPORTIVAS}

Def: Llamaremos "funciones deportivas" cada uno de los deportes federados o reconocidos internacionalmente.

A continuación hacemos un esquema de clasificación de los deportes:

\section{CLASIFICACION}

Desde el punto de vista pedagógico no todos tienen el mismo valor ni todos pueden ser practicados por todo el mundo. Quizá un programa piloto se podría hacer únicamente para los deportes que tienen posibilidad de practicarse por grandes masas. El esquí acuático puede ser deporte muy interesante como métadoppara mejorar el equilibrio, adquirir flexibilidad, tener reflejos y fuerza muscular, etc., pero no es asequible más que a muy contadas personas. No todos tienen una motora ni tampoco un mar o un lago cerca, ni apetece realizarse durante todo el año. En las variables deportivas hemos señalado y tenido en cuenta las condiciones económicas del individuo. Hay muchísimos deportes en los que el dinero juego también su parte.

En la ficha deportiva que hemos hecho se ha tenido todo en cuenta. Vds. pueden señalar los factores o variables deportivas que tal vez hemos pasado por alto.

Así y desde un punto de vista meramente pedagógico escogemos:

\section{En Atletismo:}

Carreras

de velocidad

de medio

de fondo

Con acepciones de vallas y campo a través.

$\begin{array}{ll}\text { Saltos } & \begin{array}{l}\text { de longitud } \\ \text { de altura } \\ \text { de triple } \\ \text { de pértiga } \\ \text { de peso } \\ \text { de jabalina } \\ \text { de disco }\end{array}\end{array}$

Marcha

Baloncesto-manibasket

Balonmano-balonmano alevin

Voleybol-minivoley

Esgrima florete

Esqui

Futbol

Gimnasia deportiva

Golf

Hipica

Hockey hierba y patines
Lucha-Judo

Natación-crowl, braza, españda y "delfin"

Saltos de trampolín

Remo-piragüismo

Rugby

Tenis

Tenis de mesa

Tiro con arco

\section{VARIABLES DEPORTIVAS}

Def: Llamaremos "variable deportiva" cada uno de los factores que puede influir en el rendimiento de un deporte.

Como veremos el número de variables puede ser infinito. Hemos intentado hacer una clasificación de esas variables. De seguro que no es completa, como no lo son los múltiples test que en diferentes países se vienen practicando, pero que nos sirven para marcar la pauta de actuación sistemática.

Condiciones antropométricas.

Condiciones analíticas

Condiciones fisiológicas

Valores en reposo

Cardiocirculatorio

Respiratorio

Hemático

Pruebas de esfuerzo.

Ruffier

Tuttle

Schneider

Harvard

Metabograma

Condición motora

Parámetros de forma fisica.

Resistencia y endurecimiento

Flexibilidad

Fuerza

Resistencia muscular localizada

P. habilidad motora

Coordinación neuromuscular

Agilidad

Tiempo de reacción

Explosión

Velocidad

Condición nerviosa y psicosensorial

Sexo

Edad de iniciación

Edad de competición

Planificación económica

Instalaciones

Material

Estudio económico

Antes de los 6 años 
de 6 a 8

8 a 10

10 a 12

12 a 14

14 a 18

Más de 18

madurez

senectud

La clasificación anterior afecta a las cualidades humanas del individuo. Desde el punto de vista matemático hacemos la siguiente clasificación.

\section{1) Variables lineales:}

Son variables lineales las que en la ecuación $D=F(X)$ intervienen según la primera potencia. La valoración de esta cualidad depende de cada deporte.

Por ejemplo en el futbol la resistencia puede ser una variables lineal.

\section{2) Variables cuadráticas:}

Son variable cuadráticas las que con la ecuación $D=F$ $(X)$ intervienen según la segunda potencia. La velocidad en el resultado de una carrera puede depender del cuadrado de la velocidad.

Esta classificación desde el punto de vista de nuestros fines es esencial. Nos permite de una manera global ver el valor que puede tener ya la función deporte.

El estudio de la influencia de cada variable en esa fórmula ideal que tratamos de hallar será trabajo específico de su intervención en cada deporte. Se requiere un estudio profundo de cada unos de los factores asi como du su interdependencia y correlación.

Según esta interdependencia podemos a su vez clasificar las variables asi:

\section{1) Variables independientes.}

Son aquellas que prácticamente no entran relacionadas con otras en la ecuación.

\section{2) Variables correlacionadas.}

Son aquellas variables que están intimamente relacionadas con otras.

Así el peso y velocidad en las carreras, la coordinación y la agilidad con la resistencia en los deportes de equipo.

El endurecimiento y la resistencia en las carreras de fondo o larga distancia.
El la literatura deportiva son muy empleados los ínidices. Estos son en realidad algunos de los monomios de la ecuación del deporte. Indices que por otra parte relacionan variables muy interdependientes. Para obtener la ecuación tendremos presente estos valores parciales que se ha comprobado y experimentado muchas veces. Para nuestra ecuación podemos calificar de 1 a 10 puntos cada uno de las variables en las que no haya otra medida ya tradicional. Tal sucede en el peso, talla, dinamómetria, escapular, lumbar, de empuje, etc., que tiene una unidad conocida. En realidad cada variable es una magnitud. Nuestra primera ocupación será elegir la magnitud-Unidad más adecuada para muestros fines. "Las magnitudes desempeñan un papel esencial en todas las ciencias, hasta el punto que se podria bablar del grado de desarrollo de una ciencia por el grado de elaboración alcanzado por su teoría de magnitudes correspondientes". (P. Abellanas). Para nosotros el estudio de las variables deportivas constituye, pues el núcleo de la futura investigación. Es necesario elaborar métodos de medida adecuados para cada una de éllas con relación a cada uno de los deportes, y su realización debe hacerse por especialistas. El médico, el psicólogo y el sociólogo deben trabajar juntamente en las pruebas, en las comprobaciones, en la valuación de los resultados.

Todos los resultados con su puntación se ponen en la FICHA ME DICO DEPORTIVA.

Los datos, luego se pasan a unas fichas perforadas, o a cinta magntica e incluso se pueden leer directamente por una lectora ótica. Estos son los métodos corrientes para introducir datos en una computadora tendiéndose a emplear sobre todo el último por la economía que supone en tiempo, en trabajo y también en errcres.

En la elaboración del programa para el IBM se tendrán presente todas las ventajas e inconvenientes y la viabilidad de cada uno de los métodos.

\section{UTILIDAD Y APLICACIONES.}

Tenemos ya nuestra ficha deportiva, es decir, desde el punto de la computadora nuestra tarjeta de datos.

Tenemos también nuestro programa. ¿Pero qué es lo que vamos a obtener?. Los fines que nos hemos propuesto y que salen de la computadora como resultados escritos son:

1) Clasificaión de un conjunto de FICHAS DEPORTIVAS según sus posibilidades, dadas en tanto por ciento, para cada uno de los deportes. Las fichas vienen ordenadas de mayor a menor capacidad.

2) Dada una FICHA DEPORTIVA ordenar los deportes, de mayor a menor según la posibilidad 
y capacidad del individuo para su práctica, en cada uno de los parámetros componentes.

3) Distribución de los alumnos de los centros de enseñanza media superior y laborales o bien clubs, grupos o entidades deportivas, según capacitación para los deportes a través de las fichas de aptitud. El manejo de estos miles de datos solo es posible en un ordenador electrónico.

4) Dadas unas variables deportivas de un individuo indicar el deporte o los deportes que deben realizar para alcanzar otras variables en el grado que se indique.

Esto resulta de suma importancia cuando el deporte se considera también como terapéutica en el desarrollo orgańico $y$ armónico del hombre.

5) Dado un conjunto de individuos con unas características similares, indicar los deportes que deben realizar o que más conviene practicar.

6) Hallar los deportes ideales para un individuo ideal. Me refiero en este apartado al hecho de que se puede fijar de antemano unas cualidades o variables deportivas y ver que deporte es recomendable en esta situación por la experiencia adquirada.

7) Fijado un rendimiento en un deporte y las variables naturales del individuo hallar la optimización de las restantes para que se pueda alcanzar ese tope.
Este punto resulta sugestivo ya que el desarrollo integral del individuo es nuestra primera meta.

Complemento de lo anterior es lo siguiente:

8) Estudio de los deportes como indicación educativa habida cuenta de las condiciones de todo tipo en el ambiente y circuns tancias en que se pueden practicar.

9) Selección deportiva, al menos desde un punto de vista teorico habida cuenta de que tendremos datos de la capcidad máxima, de las mejores, en los parámetros necesarios para la realización del gesto deportivo.

10) Indicaciones especificas de los deportes como terapéutica en aquellas personas que tengan disminuida parcialmente capcidades de realización, tanto como complemento, como de ocupación terapéutica de la actividad.

11) $Y$ por último, habida cuenta de los datos, se puede organizar una auténtica campaña de prevención del accidente y la enfermedad por el deporte, pues de todos resulta conocido como el vencimiento no es solo factor de la oportunidad de realización sinó de la capacidad mesurable suficiente, prevista e integralmente programada

Estos son los principales fines que la utilización de un programa en el IBM nos alcanza. En posterior estudio de estas ideas de seguro que aparecerán nueves aplicaciones, ya que el campo de una computadora es ilimitado $y$ capaz de manejar cientos de miles de datos en tiempo minimo.

\section{RADIO AID FOR COACHING A DEAF TENNIS PLAYER}

Suzan LIVINGSTON and J. G. P. WILLIAMS, F.R.C.S.

Beaconsfield School of Lawn Tennis, Beaconsfield, Bucks.

\begin{abstract}
Great difficulty has been experienced in coaching a good tennis player with a hearing loss of 100 decibals in each ear.
\end{abstract}

Use of radio aid "Russaid" has facilitated coaching very considerably, particularly after certain operating difficulties were overcome.

The possibilities of this equipment in other countries is indicated. 


\title{
THE GRAPHICAL DISPLAY OF ANGULAR MOVEMENT OF THE BODY
}

\author{
P. R. CAVANAGH, B.Ed., Ph.D.," and D. W. GRIEVE, M.Sc., Ph.D. \\ Royal Free Hospital School of Medicine, 8 Hunter Street, London, W. C. 1. \\ * Present address: Biomechanic Laboratory, College of Health \& Physical Education, \\ Pennsylvania State University, University Park, Pennsy/vania 16802, U.S.A.
}

\begin{abstract}
A display of recorded angular movements of the body should convey the temporal variations of posture at chosen joints and the relationship between them. The information should be in quantitative form and it should be possible to easily discern small changes in the patterns of movement with respect to angulation and timing. Photographic sequences, stick diagrams and angle-time diagrams partially meet these requirements.

This paper discusses an additional presentation of angular movement, the angle-angle diagram, which shows the relationship between two chosen angles throughout movement. Usually, but not necessarily, the angles refer to adjacent joints or segments of the body. In locomotion, the shapes of the patterns obtained are sensitive to small changes of performance and have found application in the clinical examination of gait. It is likely that the angle-angle diagram would be of value in the examination of movements in sport. Examples are given of patterns obtained from walking and running, stair climbing, box stepping, sit to stand movements and vertical jumping. The biomechanical purpose of the display will affect the choice of type. The interpretation and usefulness of the angle-angle diagrams and the means of obtaining them are discussed.
\end{abstract}

\section{Introduction}

The teaching and coaching of movement skills has grown up alongside the development of cine-film techniques. Cine-film and now closed circuit television provide excellent means of recording movement, and they have been welcomed by the coach in his difficult task of communicating to his pupil. The recording allows
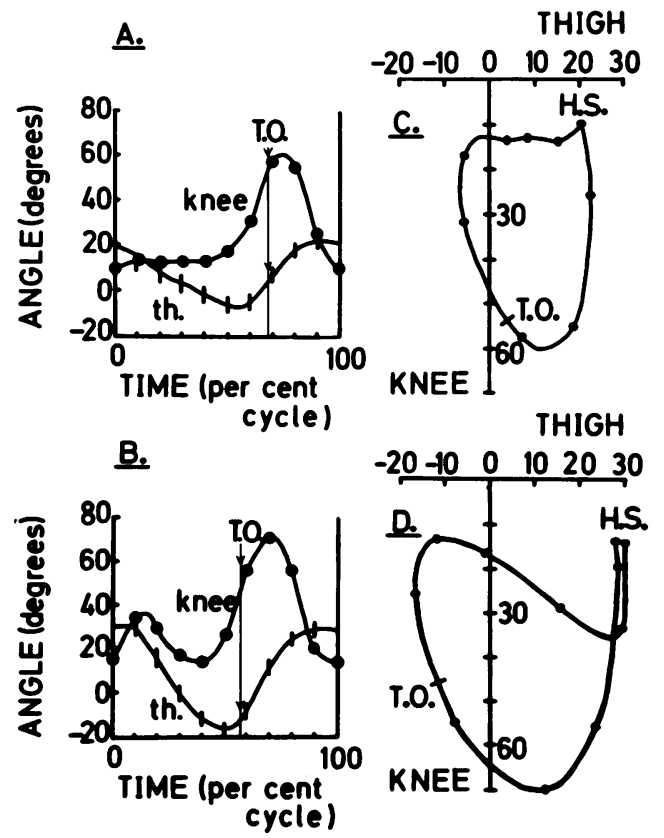

him to relive the event in its original rhythm or to slow it down. Despite these technical advances, much slower progress has been made in the interpretation and means of communicating what is to be seen on the screen. Although film contains all the information that might be required it does not do what we would like it to, that is to emphasise the things that we should be looking at. We must be very gifted, even in very simple movements, to perceive the coordinated actions involved. The desired emphasis is usually obtained by making a series of tracings from the film or by constructing stick diagrams which highlight the required details of posture. Unfortunately the eye is insensitive to differences of movement patterns shown in stick figures, and the stick figures cannot be read quantitatively. This method of communication is simply inadequate for the purpose and we must think of an alternative approach.

What is needed is a diagram whose shape is sensitive to details of the movement, which can be judged qualitatively or quantitively, depending upon the user. At one level, the shape itself could be used by the coach as an indication of good or bad performance, while at another level the same diagram would convey accurate quantitative information for further analysis. A common graphical approach is to measure the limb angles from film and plot them against time, as is done in diagrams $A$ and $B$ of figure 1 which describe walking. Cycle time from heel strike is plotted along the $x$-axis and degrees of rotation in the sagittal plane are plotted along the $y$-axis. We can follow from these graphs the time course of the thigh angle and knee angle. It is difficult to obtain information from the diagram about the coordinated 
movements of the limb as a whole and to visualise its posture. Figure $1 \mathrm{~A}$ describes the pattern of movement of someone walking very slowly. When we walk faster, the movements at the thigh and knee change both in amplitude and in their relation to each other. The new pattern is recorded in figure $1 \mathrm{~B}$. Substantial differences exist between the patterns of movement but the eye is not very sensitive to the important differences between them. Diagrams $A$ and $B$ in figure 1 emphasise (one could say overemphasise) the relationships of the separate joint movements to time. We think that a diagram which emphasises the relationship of the joint movements to each other is a more suitable way of presenting the patterns of movement and is easier to interpret.

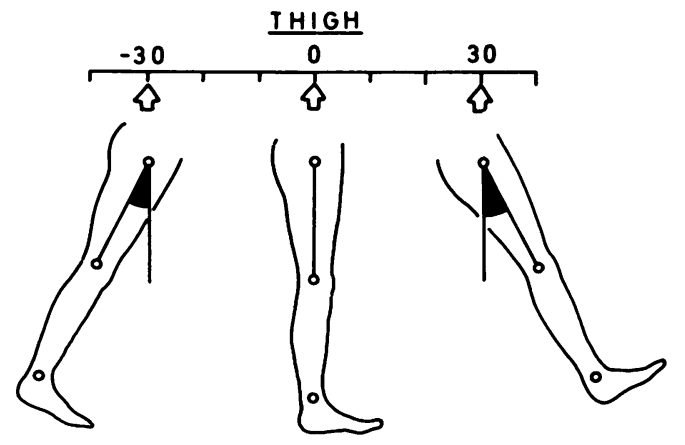

Instead of plotting an angle against time we plot it against another angle. This is usually, but not necessarily, the angle of an adjacent limb segment. We shall now develop a diagram in which the angle of the thigh to the vertical is plotted against the angle of the knee joint. Let us do so in easy stages before referring back to Figure 1. Figure 2 shows three positions of a thigh. Points on a horizontal scale are used to represent the angle that the thigh makes with the vertical. We adopt a convention that movement of the thigh in front of the vertical is measured as a positive and movement behind the vertical is measured as a negative angle. Incidentally, we are talking about the inclination of the thigh to the vertical and not the posture of the hip joint. Figure 3 shows three postures of a knee joint. The knee angle is that between the long axis of the thigh and the long axis of the shank. In Fig. 3, as in subsequent charts, we represent the knee angle on a vertical scale. Knee angles of 9,47 and 88 degrees have been represented.

Figure 4 shows a girl at four instants in a standing broad jump. The two scales representing the thigh and knee angles are used as the axes of a chart that we call the THIGH/KNEE diagram. A point on this chart represents both a thigh angle and a knee angle. The points shown on the chart thus represent instantaneous postures of the limb during the jump. Looking first at posture (a) at the beginning of the jump, the thigh angle

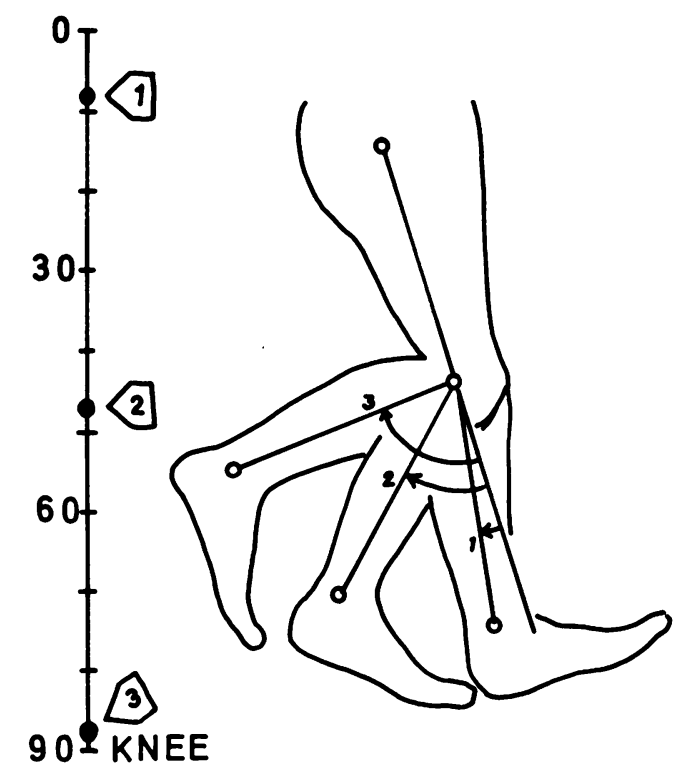

of 22 degrees and knee angle of $\mathbf{4 6}$ degrees are plotted together at point (a) on the chart to represent the posture of the whole limb. Postures (b), (c) and (d) are represented by combinations of thigh and knee angles of $(0,61),(63,54)$ and $(111,121)$ respectively. From this movement only four distinct stages of the jump are represented on the THIGH/KNEE diagram. If all the intermediate stages had been included the curve obtained would tell us the complete pattern of movement of both the thigh and the knee throughout the jump. Thus we have a method of indicating both posture and changes of posture in a compact form.

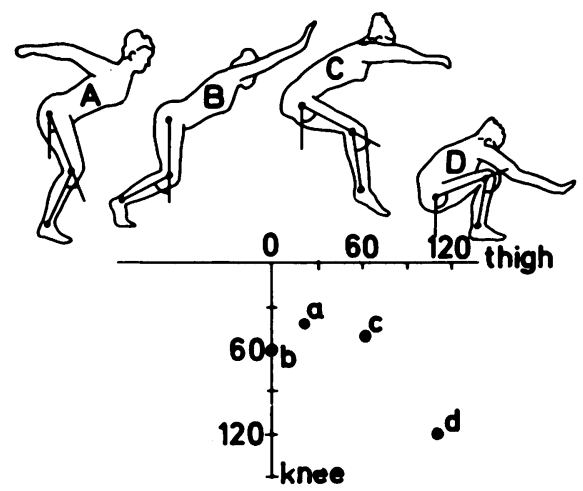

Now turn back to Figure 1. The THIGH/KNEE diagrams (Figs. $1 \mathrm{C}$ and $1 \mathrm{D}$ ) on the right contain the same information as the ANGLE/TIME diagrams on the left. The differences in the coordinated patterns of movement are more readily detected in the angle/angle diagrams. The thigh-knee diagrams are sensitive to changes in the speed of walking. Figure 5 shows the 


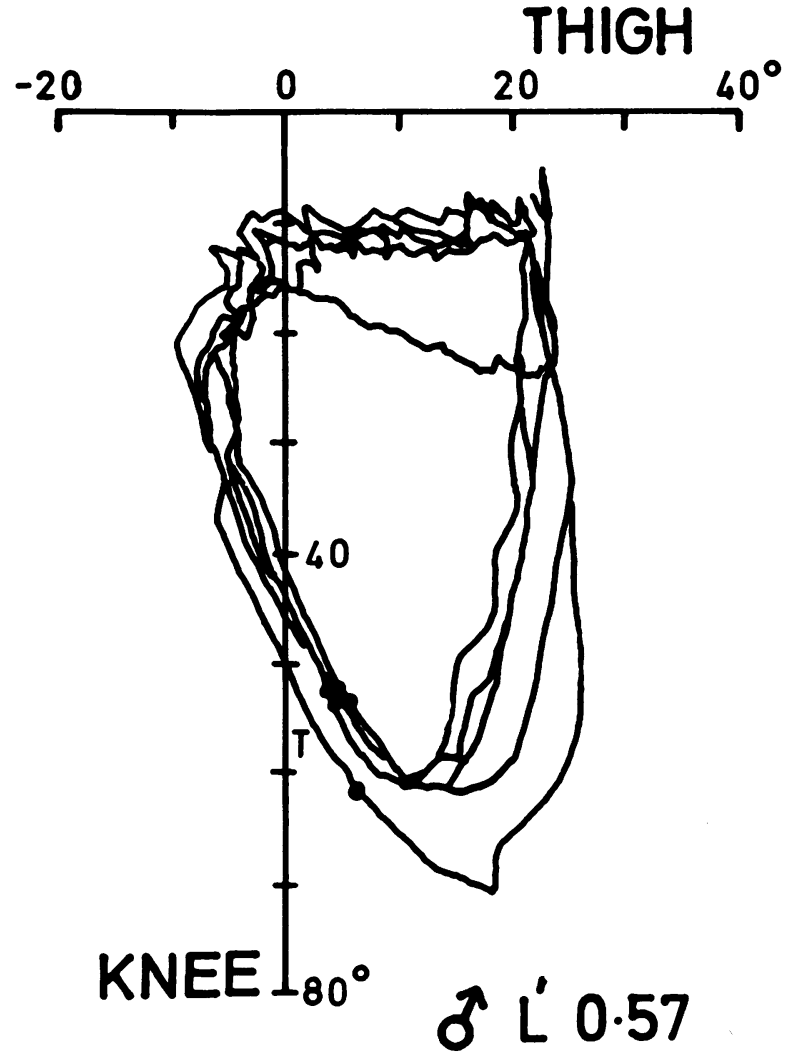

curves for five subjects walking at the same speed. The graphs contain all the angular data of the two limb segments which can be read quantitatively. Alternatively a coach may find it adequate to work with the shapes alone, once he has familiarised himself with the patterns associated with different types of performance. If we compare the shape of this family of curves with that found in Figure 6 we see immediately that something has happened to change the shape which means that the pattern of movement has changed. The new shape is a characteristic one obtained when walking at a higher speed. The loops are larger and more rounded. From these diagrams it is easy to pick out the coordinated actions of adjacent parts of the body. For example, reading the diagram anti-clockwise, the vertical descent of the curve from heel strike (top right) tells us that the thigh angle remains fixed while the knee flexes through 20 degrees. We also see a coordinated straightening of the knee with extension of the thigh at the hip. These features were absent at the low speeds of walking shown in Fig. 5. These two sets of thigh/knee diagrams cover the same range of speeds as did the upper and lower diagrams in Fig. 1. The differences between the two patterns of movement are much clearer in the angle-angle diagrams.

To complete the description of the lower limb, the ankle has to be considered. Figure 7 shows an ANKLE/KNEE diagram taken from walking. The knee angle is represented in the same way as before. Knee flexion moves a point on the graph downwards, knee extension moves it upwards. We have replaced the thigh angle on the $X$-axis by an ankle angle. An ankle angle of 90 degrees is what you would get standing on the ground with a vertical shank. Plantar flexion reduces this angle to the left of the diagram and dorsiflexion increases it to the right. The heel strikes the ground at the point marked $H$. This is followed by a period of coordinated knee flexion and slight plantar flexion at the ankle. The diagram moves downwards and to the left. Single support then begins. The diagram moves upwards and to the right, indicating that the knee is extending while dorsiflexion is taking place. Plantar flexion then occurs and the knee flexes. At toe off, shown as $T$ on the diagram, the knee continues to flex. As the limb curls up, the ankle is dorsiflexed to clear the toes from the ground and the knee extends through to heel strike.

It is very difficult to make interpretations of this nature from movie film or from angle-time diagrams. It is quite easy to see from the ankle/knee diagrams how the coordination between knee and ankle changes dramatically with the speed of walking. It is worth

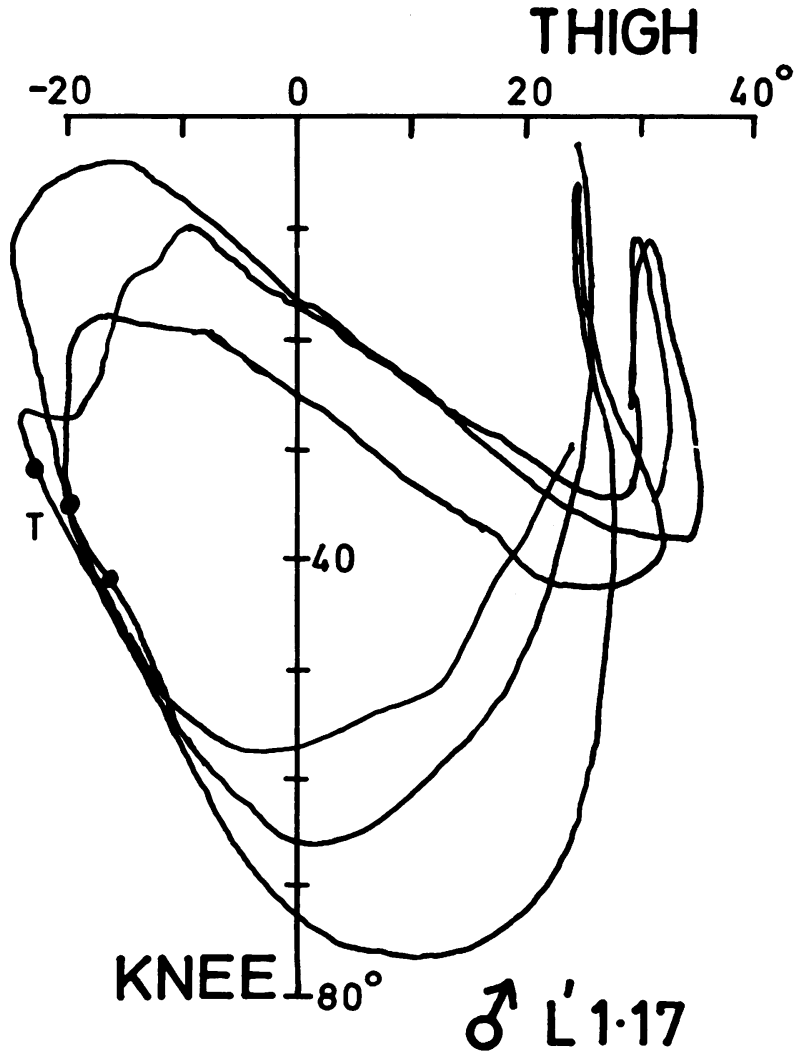


noting that we have not sacrificed time information in angle-angle diagrams. The dots along the curve represent the time scale, in this case tenths of the total walking cycle time. The double lines indicate periods of double support.

We recently became interested in why race walkers as a group suffer from excessive shin pain. A possible reason for this pain which ordinary fast walkers do not experience is that the pre-tibial muscles are being used excessively. We obtained electromyographic evidence that this was the case and then turned to angle-angle diagrams to see what mechanical insults were being applied to the muscle group. Figure 8 shows the THIGH/KNEE and ANKLE/KNEE diagrams of Colin Young, an International race walker, walking at top speed. The international rules state that the leg must be straight at some stage during contact with the ground. The diagram shows that the knee was indeed held

\section{plantar ANKLE dorsi}

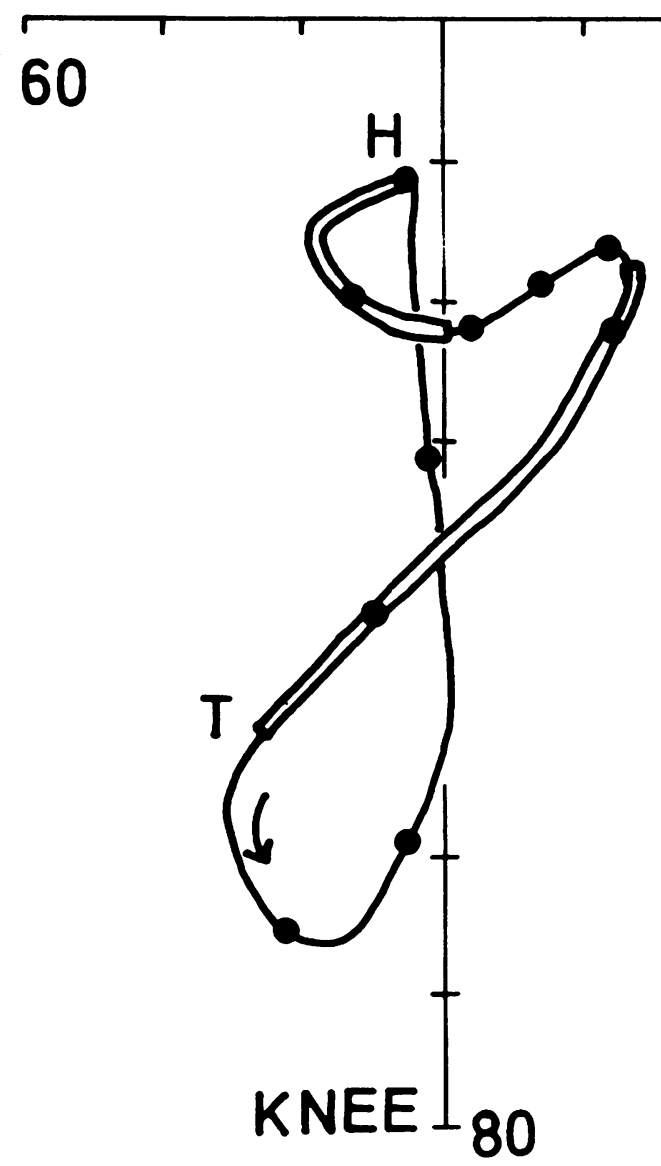

straight for more than half of the stance phase, quite unlike ordinary fast walking. This means that the impact of heel strike is transmitted to the ankle without knee cushioning. The ankle-knee diagram shows that the anterior tibial muscles which are active at this stage are forcibly stretched by $\mathbf{2 0}$ degrees of plantar flexion in this period following heel strike.
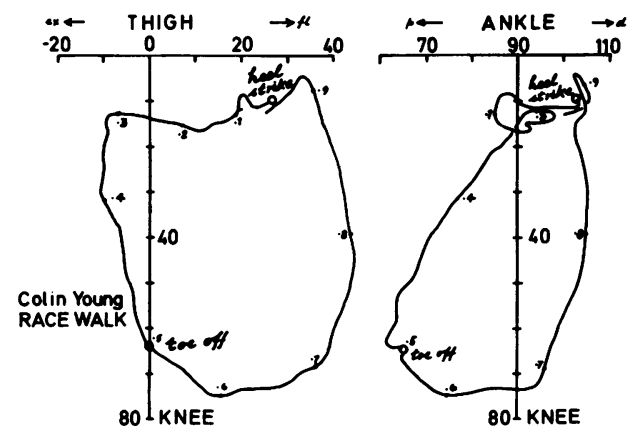

Let us now look at a different movement, stair climbing, and show how additional information can be presented on an angle-angle diagram. Figure 9 shows the THIGH-KNEE diagram describing the pattern of movement involved in stair climbing. The dotted line shows the pattern of movement when ascending one eight inch stair, the solid line the pattern for ascending two eight inch stairs at a time. These patterns are seen to be quite similar in character, two steps requiring only an? increase in the amounts of thigh and knee flexion. The third scale on this chart shows the angle of the shank to

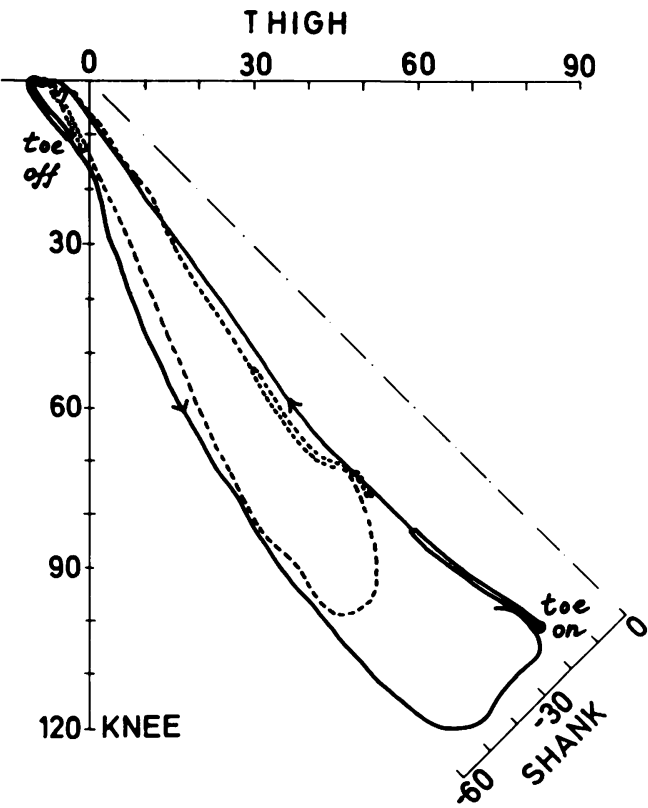

STAIR CLIMBING 
the vertical. The shank angle can be represented because it is a function solely of thigh angle and knee angle. We can read the shank angle obliquely on the scale at any stage in the movement. We see that after the toe strikes the stair, there is a period of knee extension and thigh extension. Reference to the third scale shows that this knee extension occurs about an almost fixed shank, the shank moving only ten degrees while one hundred degrees of knee extension if occurring. That is to say, the knee is fixed in space and we lever ourselves up over it. We can also see that the shank trails behind during the swing through, finally straightening twenty degrees for placement. If we know the thigh and knee angles, we can work out where the ankle is in space relative to the hip joint. The user of the thigh-knee diagram has no need to make this calculation because the information can be read directly from the diagram, as is shown in Fig. 10.

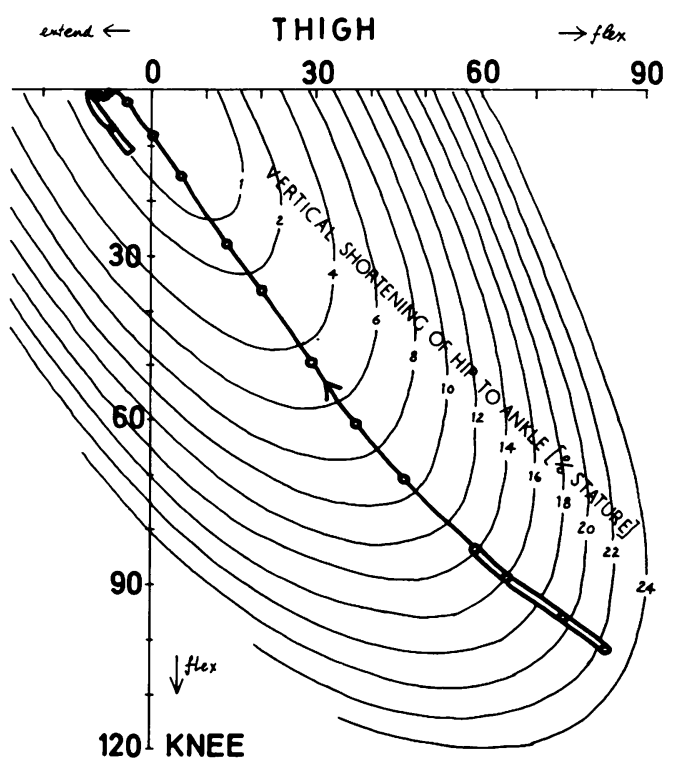

Gaining height in stair-climbing.

Suppose we wish to know the contributions that the thigh and knee make to the total gain of height during the stair climbing movement. Figure 10 is another thigh-knee diagram. In addition to the thigh and knee axes the chart contains lines of equal height of the hip above the ankle calculated from anthropometric data. Any combination of thigh and knee angles along a 'line of constant height' represents a particular reduction from the maximal vertical distance that is possible between hip and ankle. Not only can we read off from this graph the fact that $22 \%$ of stature has been gained during the movement in support, but also we see that of all the possible ways of gaining height the chosen pattern of movement achieves it in the most direct manner, cutting the lines of constant height perpendicularly.

In many movements in sport, the inertia of parts of the body affect performance. The inertia of the lower limb depends only upon the postures of the knee and ankle. Figure 11 shows how the moment of inertia about the hip can be read off directly from an ANKLE/KNEE diagram. Such a diagram might show the coach how a change in the posture would affect the inertia. The ankle axis appears as usual - plantar flexion to the left, dorsiflexion to the right, as does the knee axis with extension and flexion upwards and downwards respectively. But in addition we see the moment of inertia of the swinging limb indicated by the lines crossing the diagram.

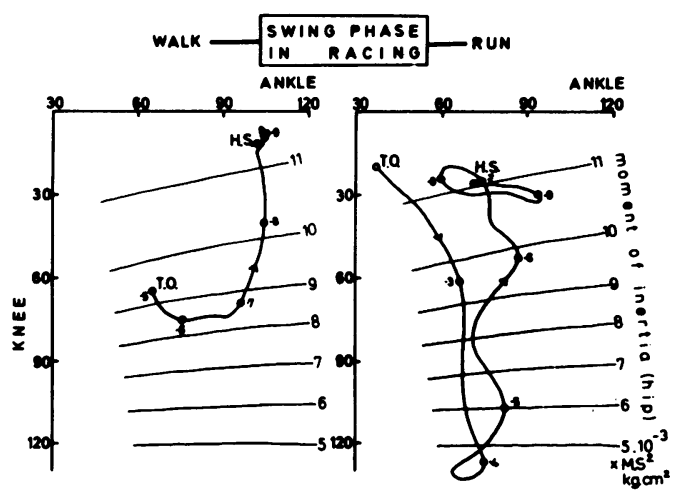

We see that flexing the knee decreases the inertia of the limb, as does dorsiflexing the foot. The two examples depict race walking and running. The considerable extra reduction of inertia of the limb that is achieved in running is readily detected.

The diagrams we have shown were all obtained from the rather tedious analysis of cine film. Television does not make this analysis any easier. The diagrams will be of real use when they can be obtained as quickly as a television recording. We envisage devices which will measure and display the required angles during performance of the movement. Electrogoniometers and polarised light goniometers represent a first generation of such devices, although in many sporting movements the technical problems of recording have not been solved. The angle-angle diagrams have proved to be a compact way of representing patterns of movement, and because they are sensitive to small changes in the patterns of movement we think that they will be useful in the study of movement in sport. 


\title{
THE PRESENT LIMITATIONS IN THE KNOWLEDGE OF THE BIOLOGY BOTH ULTRA STRUCTURAL AND FUNCTIONAL; BEFORE, DURING AND AFTER EFFORT AT DIFFERENT RATES
}

\section{LA DELIMITATION ACTUELLE DES CONNAISSANCES DE LA BIOLOGIE ULTRASTRUCTURALLE ET FONCTIONNELLE - AVANT, PENDANT ET APRES D'UNE GAMME D'INTENSITE D'EFFORT}

\author{
R. ŻUNIC, P. GALLE, V. ŽUNIC and J. P. BERRY
}

\begin{abstract}
The authors state that the current method of studying samples prepared for electron microscopy which breaks up the basic enzymes and iso enzyme systems. If these results are compared with those obtained by bio-physical analysis this confirms and supports the idea that a false picture is built up of what really happens and artefacts are created.

We have compared the values by using the techniques of electron microscopy and biophysics. Those sections preserved by fixing at $-14^{\circ} \mathrm{C}$ maintained the composition of tissues before, during and after effort at their full value. Sections prepared by current methods for ultra structure research show a complete absence of co-enzymes and the other factors operating at the atomic level.

Coupes de tissu d'epaisseur $\sim 400 \AA$ pour les recherches ultrastructuralle, preparés par la technique actuelle, montre grâce à nos analyses biophysiques comparées, l'absence de tous les facteurs au niveau atomique ( $\mathrm{Ca}, \mathrm{P}, \mathrm{Mg}, \mathrm{Zn}, \mathrm{Cu}$ et egalment $\mathrm{K}, \mathrm{Na}, \mathrm{Cl}$, comme on peut voir sur les diapositives).

La Preparation du tissu en ce moment pour les recherches structuralles au microscope électronique chasse cofacteurs primordiales d'enzymes où d'isoenzymes provoquant des alterations profondes non seulement au niveau moléculaire. Ces alterations n'épargne aucunement la structure intracellulaire: toutes les organites sont ateintes. La membrane cellulaire dans ces conditions même sill n'ai pas alteré, ne peut avoir que l'aspect du sens unilateral-du milieu intracellulaire au milieu extracellulaire dans sa fonction.

D'après nos analyses biophysiques sur les coupes du tissu pour le microscope electronique, nous avons les perturbations du milieu extracellulaire, lesquelles ne

facilitent pas les recherches, au contraire, deforment les conditions biologiques d'étude de l'espace intercellulaire (des neurones).

La technique de la preparation des coupes, non seulement que reduit l'aspect fonctionel d'ultrastructure, mais en même temps, dans la même proportion crée d'arte fact, sans tenir compte des consequence de la? conversion des processus physicochimiques incopatibles biologiquement.

Nous avons comparé les valeurs des techniques de microscope electronique et de biophysique relationnelle (les coups 40 - 50 fois plus epais $=\sim 2 \gamma$ ) par la recherche simultané sur $1 \gamma^{3}$. Les coupes congelées immediatement in situ à $\geqslant 140^{\circ} \mathrm{C}$ fixent et conservent les composants du tissu avant, pendant et après l'effort dans sa valeur integrale. Les coupes de la preparation actuelle pour la recherche ultrastructuralle montre absence total des coenzymes et les autres effecteurs au niveau atomique.
\end{abstract}




\title{
A NEW TECHNIQUE FOR OBJECTIVE EVALUATION OF MUSCULAR EFFORT DURING EXERCISE
}

\author{
RABINDRA NATH SEN*, R. H. HARDING \& P. R. M. JONES \\ Department of Ergonomics \& Cybernetics, University of Technology, Loughborough, Leics. \\ *Visiting University Research Fellow on leave from Industrial Physiology \\ Division, Central Labour Institute, Sion, Bombay, India
}

\begin{abstract}
In athletic activities it is desirable to have information about i) the extent to which different muscle groups of the body take part, ii) the degree of improvement in a training programme, iii) the difference in the muscular efficiency in different individuals, iv) the effect of fatigue. The information from parameters such as heart rate, oxygen consumption, biochemical substances in body fluids, etc., may be sufficient, but these are indirect measurements of muscular activity and have certain other disadvantages.
\end{abstract}

The action potentials of individual muscles, electromyograms (EMGs), directly reflect the qualitative aspect of the muscular activity. An attempt to obtain a quantitative picture by the integration of the surface recorded EMG after full-wave rectification involves a large number of electrodes with a corresponding number of channels and makes the technique unwieldy and unsuitable for use in the field.

A new technique to evaluate muscular activity of a particular region or different regions of the body by quantifying the EMG picked up'by several sets of bi-unipolar surface electrodes (i.e. one unipolar over flexors and another over extensors) after passing through a summing amplifier was tried on a group of subjects at different resting postures as well as during exercise.

The results indicate that this technique could be used with advantage in athletic activities in addition to the methods for other physiological parameters.

\section{SEQUENTIAL TIMING OF OVERHAND PATTERNS OF WOMEN ATHLETES}

\author{
M. J. ADRIAN and M. L. ENBERG \\ Department of Physical Education for Women, Washington State University, Pullman, Washington 99163
}

\section{ABSTRACT}

Competitive badminton, volleyball and tennis players utilise overhand patterns which are considered similar in nature. Coaches often relate one pattern to another and assume that the mastery of one pattern facilitates the learning of another. However, this assumption may be false and may lead to interference with the expert performance of the other patterns. Therefore, this investigation was conducted to identify similarities and differences within and among competitive women players of three sports. One subject (DC) had competed on the intercollegiate level in all three sports. The second subject (KB) competed in tennis since childhood and the third subject $(\mathrm{CH})$ was an international badminton player.

All subjects were filmed at 730-775 frames per second, performing the badminton smash, tennis serve and overhand volleyball serve. Subject DC's performance showed greater intra-individual differences than did the other subjects. Subject BC assumed the tennis slice serve pattern for all her strokes; whereas $\mathrm{CH}^{\prime}$ 's patterns resembled her badminton stroke.

Two basic patterns were noted. One pattern involved a step forward followed by pelvic rotation and the classic "unwinding" sequences for force production. The other pattern introduced flexion in the anteroposterior plane, as a hip-trunk and/or knee-trunk action.

The results lead one to question whether the patterns resulted from individual structural difference, order of learning or whether, in fact, one pattern is better suited to the performance of a given skill. 


\title{
PRESENTING AN APPARATUS FOR HOLDING THE LOWER LIMBS STEADY DURING X-RAY, TO CHECK AND MEASURE DISCREPANCIES OF LIMBS AND PELVIS, AS WELL AS TO SUPPLY A STEADY, ACCURATE AND REPRODUCIBLE BASIS FOR LATERAL DEVIATIONS OF THE VERTEBRAL COLUMN
}

\author{
A. DAL MONTE, M.D. \\ Cattedra di Medicina dello Sport dell'Universita di Roma \\ C.O.N.I. - Istituto di Medicina dello Sport
}

\begin{abstract}
The aim of this apparatus is to afford a standard method to $X$-ray the differences in length of lower limbs, of pelvic discrepancies, and to supply a steady, accurate and reproducible basis to assess lateral deviations of the vertebral column.

These radiographic problems have been noted in hospitals as well, several instruments having been made. As regards sports medicine, standardizing radiographic data is even more important, because in most cases the phenomena to be evaluated are less obvious than in hospital patients. Hence, it is necessary to improve research techniques to be able to evaluate them.

The apparatus that we made consists of a stiff and levelled platform. On it is a panel, fastened to a rotating axis which is placed between the lower limbs of the subject under examination.The feet of the latter rest on two stiff boards that can slide vertically, by means of an electric elevator. The panel or baffle that is placed between the legs can be brought up nearly to touch the perineum of the subject, by means of the vertical motion of the boards. Because of the vertical movement of the boards, very young children can also be evaluated.
\end{abstract}

By placing the subject astride on the baffle, and making the thighs and legs adhere to the stiff baffle, and by placing the feet in the correct position on the footboards, we fix the geometric system with vertices at the two feet and the two coxo-femoral joints. It is thus possible to achieve complete pelvic control.

In this way we do away with all postural adjustments that the subject with possible limb anomalies or deviations of the vertebral column, may have acquired in time.

X-rays of the pelvis and of the lower limbs, antero-posterior projection, makes it possible to measure exactly the differences in length of the limbs and pelvic discrepancies, as well as assessing accurately the degree of lateral deviation of the vertebral column.

Should the lower limbs differ in length, the exact range of the anomaly can be found out by raising the lower limb with objects the thickness of which is known.

The apparatus can be caused to rotate on 3600 , if it is necessary to make X-rays with lateral and oblique projections. 